\title{
Base polytopes of series-parallel posets: Linear description and optimization
}

\author{
Rainer Schrader ${ }^{a, *}$, Andreas S. Schulz ${ }^{b}$, Georg Wambach ${ }^{\text {a }}$ \\ a Institut für Informatik und Zentrum für Paralleles Rechnen, Universität zu Köln, Weyertal 86-90, \\ D-5093I Köln, Germany

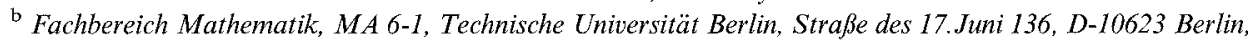 \\ Germany
}

Received 20 June 1995; accepted 10 June 1997

\begin{abstract}
We define the base polytope $B(P, g)$ of a partially ordered set $P$ and a supermodular function $g$ on the ideals of $P$ as the convex hull of the incidence vectors of all linear extensions of $P$. This new class of polytopes contains, among others, the base polytopes of supermodular systems and permutahedra as special cases. After introducing the notion of compatibility for $g$, we give a complete linear description of $B(P, g)$ for series-parallel posets and compatible functions $g$. In addition, we describe a greedy-type procedure which exhibits Sidney's job sequencing algorithm to minimize the total weighted completion time as a natural extension of the matroidal greedy algorithm from sets to posets. (C) 1998 The Mathematical Programing Society, Inc. Published by Elsevier Science B.V.
\end{abstract}

Keywords: Polyhedral combinatorics; Series-parallel posets; Base polytopes; Supermodular functions; Greedy algorithm; Integer programming

\section{Introduction}

More than twenty years ago, Sidney [1] published his article on the minimization of the weighted sum of completion times for one-machine scheduling problems with precedence constraints. The algorithm he proposed generalizes Smith's greedy-type rule for independent jobs [2]. Sidney proved that there always is an optimal schedule starting with an ideal of maximum weight density. Hence, the problem can be solved recursively for this ideal and its complement.

While in general the only ideal with maximum weight density may be the whole set, and so nothing is gained, Sidney showed that in the case of series-parallel precedence constraints, we can always find a proper sub-ideal to start with. Sidney's al-

\footnotetext{
${ }^{*}$ Corresponding author. E-mail: rs@informatik.uni-koeln.de.
} 
gorithm is a greedy algorithm extended from sets to special partially ordered sets. However, the precise relation of this algorithm to structures induced by sub- or supermodular functions, which many people would consider the natural setting for greedy algorithms, remained unclear. The purpose of this paper is to shed some light on this relation.

We follow a common approach and study polyhedra induced by supermodular functions (the interested reader is referred to Fujishige's book [3] and the references cited therein). It is well known that the linear minimization problem over the base polytope $B(g)=\left\{x \in \mathbb{R}^{E}: x(S)=\sum_{e \in S} x_{e} \geqslant g(S)\right.$ for all $\left.S \subset E, x(E)=g(E)\right\}$ of a supermodular function $g$ can be solved by the greedy algorithm. Every permutation $L=e_{1} \ldots e_{n}$ of $E$ induces by $x_{e_{i}}=g\left(\left\{e_{1}, \ldots, e_{i}\right\}\right)-g\left(\left\{e_{1}, \ldots, e_{i-1}\right\}\right)$ a vertex of $B(g)$, and every vertex of the base polytope can be obtained in this way. While supermodular functions $g$ acting on families of subsets of a finite set seem to be well understood, at least as far as minimization of linear functions on $B(g)$ is concerned, much less is known if, in addition, the solutions have to respect a partial order relation. Let $P$ be a partially ordered set and $g$ a supermodular function on the ideals of $P$. To every linear extension of $P$, i.e., every permutation of $P$ which respects the partial ordering, we associate an incidence vector in the very same way as for $B(g)$. The base polytope $B(P, g)$ of $P$ is the convex hull of the incidence vectors of all linear extensions of $P$. In Section 2 of this paper we introduce the notion of compatibility for a supermodular function $g$ on the ideals of $P$. In Section 3, we discuss some geometric properties of base polytopes of arbitrary posets, whereas in Section 4 we derive a complete linear description of $B(P, g)$ for series -parallel posets and supermodular and compatible functions $g$. Section 5 describes a greedy algorithm to minimize linear functions over $B(P, g)$. Instead of repeatedly picking elements with large weights, as is done in the matroid case, it follows Sidney's recipe [1] and chooses ideals with large average weight. Section 6 gives some examples and extensions.

\section{Notations and definitions}

Let $P=(E,<)$ be a partially ordered set (poset) on a finite set $E$ with $n$ elements. A linear extension $L=e_{1} e_{2} \ldots e_{n}$ of $P$ is a total ordering of the elements of $E$ which respects the partial ordering of $P$, i.e., $e_{i}<e_{j}$ in $P$ implies $i<j$. By $\mathscr{L}(P)$ we denote the set of all linear extensions of $P$.

Given two disjoint posets $\left(P_{1},<_{1}\right)$ and $\left(P_{2},<_{2}\right)$ we define two compositions on $P=P_{1} \cup P_{2}$ :

Parallel composition: $P=P_{1} \| P_{2}$,

$$
x<y \text { if }\left\{\begin{array}{l}
x<_{1} y \text { and } x, y \in P_{1}, \\
x<_{2} y \text { and } x, y \in P_{2} .
\end{array}\right.
$$


Series composition: $P=P_{1} \oplus P_{2}$,

$$
x<y \text { if }\left\{\begin{array}{l}
x<_{1} y \text { and } x, y \in P_{1}, \\
x<_{2} y \text { and } x, y \in P_{2}, \\
x \in P_{1}, y \in P_{2} .
\end{array}\right.
$$

A poset is series parallel if it can be constructed inductively from singletons by applying parallel and series compositions. Equivalently, a poset is series-parallel if no four elements induce an $N$ [4]. Four elements $a, b, c, d$ induce an $N$, if $a<b, b>c$, and $c<d$ are the only comparabilities among them.

A subset $I \subseteq P$ is an ideal if $x \in I$ and $y<x$ implies $y \in I$. In particular, $I(x)=\{y: y \leqslant x\}$ is the principal ideal induced by $x$. For $X \subseteq E$, let $I(X)=\cup(I(x): x \in X)$. By $\mathscr{I}(P)$ we denote the set of all ideals of $P . \mathscr{I}(P)$ is a lattice, i.e., for ideals $I$ and $J$ in $\mathscr{I}(P)$, also $I \cap J$ and $I \cup J$ are in $\mathscr{I}(P)$.

A convex set $C \subseteq P$ is a subset which contains with $x, y \in C$ all elements $z \in C$ satisfying $x<z<y$.

Given a lattice $\mathscr{L}$, a function $g: \mathscr{L} \rightarrow \mathbb{R} \cup\{-\infty\}$ is supermodular if

$$
g(X \cup Y)+g(X \cap Y) \geqslant g(X)+g(Y)
$$

holds for all elements $X, Y \in \mathscr{L}$. The function $g$ is called strictly supermodular if, in addition, inequality (1) holds strictly for all pairs $X, Y \in \mathscr{L}$ such that neither $X \subseteq Y$, nor $Y \subseteq X$. We will assume throughout that supermodular functions are normalized, i.e., $g(\emptyset)=0$.

Let $A, B \subseteq E$ be two disjoint subsets of $E$ (where $E$ is the ground-set of $P$ ). We call the tuple $(A, B)$ a series-reducible convex set if $A \cup B$ is convex and if $a<b$ holds for all $a \in A$ and $b \in B$. Note that if we fix one set, say $A$, then the collection of all sets $B$ which together with $A$ form a series-reducible convex set is a lattice.

Series-reducible convexity can be viewed as a kind of covering relation on certain subsets of $P$. A subset $B$ of a series-reducible convex set $(A, B)$ covers $A$ in a linear extension $L$ of $P$, if $A \cup B$ is a chain in $L$, i.e., $L=L_{I} L_{A} L_{B} L_{J}$. If we consider $A$ and $B$ as sets and do not care about individual elements, the partial ordering fixes the linear extension to proceed with $A$ first, then $B$. The impossibility of rearranging $L_{A} L_{B}$ w.r.t. $A$ and $B$ is independent of the sets $I$ and $J$. If this local independence is reflected by a function $g: \mathscr{I}(P) \rightarrow \mathbb{R}$, we call $g$ compatible (with $P$ ). More precisely, $g: \mathscr{I}(P) \rightarrow \mathbb{R}$ is compatible (with $P$ ) if for all series-reducible convex sets $(A, B)$ and any $I \in \mathscr{I}(P), I \subseteq E \backslash(A \cup B)$ such that $I \cup A \in \mathscr{I}(P)$ and $I \cup A \cup B \in \mathscr{I}(P)$, the term

$$
|A|[g(I \cup A \cup B)-g(I \cup A)]-|B|[g(I \cup A)-g(I)]
$$

is a constant $g_{A, B}$ independent of $I$. Here, we have multiplied by $|A|$ and $|B|$ to get rid of possible different cardinalities of $A$ and $B$. Any compatible function $g$ then induces a function $f$ on the series-reducible convex sets via $f(A, B)=g_{A, B}$. Modular functions 
$g$ are always compatible. Supermodular functions are always compatible with weak orders (see Section 6, where the definition of weakness and some applications in scheduling are given).

Lemma 1. Let $P$ be a poset and $g: \mathscr{I}(P) \rightarrow \mathbb{R}$ a function on the ideals of $P$. If $g$ is compatible and supermodular, then the function $f$ induced by $g$ on the series-reducible convex sets of $P$ is supermodular in both components.

Proof. (i) Let $\left(A, B_{1}\right)$ and $\left(A, B_{2}\right)$ be two series-reducible convex sets. Choose $I=I\left(A \cup B_{1} \cup B_{2}\right) \backslash\left(A \cup B_{1} \cup B_{2}\right)$. Then

$$
\begin{aligned}
& f\left(A, B_{1}\right)+f\left(A, B_{2}\right)=|A|\left[g\left(I \cup A \cup B_{1}\right)+g\left(I \cup A \cup B_{2}\right)-2 g(I \cup A)\right] \\
& -\left(\left|B_{1}\right|+\left|B_{2}\right|\right)[g(I \cup A)-g(I)] \leqslant|A|\left[g\left(I \cup A \cup\left(B_{1} \cup B_{2}\right)\right)\right. \\
& \left.+g\left(I \cup A \cup\left(B_{1} \cap B_{2}\right)\right)-2 g(I \cup A)\right]-\left(\left|B_{1}\right|+\left|B_{2}\right|\right)[g(I \cup A)-g(I)] \\
& =f\left(A, B_{1} \cup B_{2}\right)+f\left(A, B_{1} \cap B_{2}\right) .
\end{aligned}
$$

(ii) Let $\left(A_{1}, B\right)$ and $\left(A_{2}, B\right)$ be two series-reducible convex sets. Choose $I=I\left(A_{1} \cup A_{2} \cup B\right) \backslash\left(A_{1} \cup A_{2} \cup B\right)$ and let $I_{1}=I \cup\left(A_{2} \backslash A_{1}\right), I_{2}=I \cup\left(A_{1} \backslash A_{2}\right)$ and $J=I_{1} \cup I_{2}$. Observe that $I_{1} \cup A_{1}=I \cup\left(A_{1} \cup A_{2}\right)=J \cup\left(A_{1} \cap A_{2}\right)=I_{2} \cup A_{2}$. Hence

$$
\begin{aligned}
& f\left(A_{1}, B\right)+f\left(A_{2}, B\right)=\left|A_{1}\right|\left[g\left(I_{1} \cup A_{1} \cup B\right)-g\left(I_{1} \cup A_{1}\right)\right] \\
& +\left|A_{2}\right|\left[g\left(I_{2} \cup A_{2} \cup B\right)-g\left(I_{2} \cup A_{2}\right)\right]-|B|\left[g\left(I_{1} \cup A_{1}\right)+g\left(I_{2} \cup A_{2}\right)\right. \\
& \left.-g\left(I_{1}\right)-g\left(I_{2}\right)\right]=\left|A_{1} \cup A_{2}\right|\left[g\left(I \cup A_{1} \cup A_{2} \cup B\right)-g\left(I \cup A_{1} \cup A_{2}\right)\right] \\
& -|B|\left[g\left(I \cup A_{1} \cup A_{2}\right)-g\left(I_{1}\right)\right]+\left|A_{1} \cap A_{2}\right|\left[g\left(J \cup\left(A_{1} \cap A_{2}\right) \cup B\right)\right. \\
& \left.-g\left(J \cup\left(A_{1} \cap A_{2}\right)\right)\right]-|B|\left[g\left(J \cup\left(A_{1} \cap A_{2}\right)\right)-g\left(I_{2}\right)\right] \leqslant f\left(A_{1} \cup A_{2}, B\right) \\
& +f\left(A_{1} \cap A_{2}, B\right) \text { by supermodularity. }
\end{aligned}
$$

For a subset $S$ of $P$ and $x \in \mathbb{R}^{P}$, let $x(S):=\sum_{e \in S} x_{e}$. If $S=\{e\}$ is a singleton, we omit the brackets, i.e., $x(e)=x(\{e\})=x_{e}$.

\section{The base polytope of a poset}

Recall that the base polytope of a supermodular function $g$ is defined as $B(g)=\left\{x \in \mathbb{R}^{E}: x(S) \geqslant g(S)\right.$ for all $\left.S \subset E, x(E)=g(E)\right\}$. Equivalently, it is the convex hull of the incidence vectors $x(L)$ of all permutations $L=e_{1} \ldots e_{n}$ of $E$. Here, the incidence vector $x(L)$ is defined by $x_{e_{i}}=g\left(\left\{e_{1}, \ldots, e_{i}\right\}\right)-g\left(\left\{e_{1}, \ldots, e_{i-1}\right\}\right)$ for $1 \leqslant i \leqslant n$. A prominent member of the class of base polytopes is the permutahedron (cf., e.g., [5-7]). It is defined as the convex hull of all permutations, perm $=\operatorname{conv}\{(\pi(1), \ldots, \pi(n)): \pi$ permutation of $\mathrm{E}\}$. In more general terms, the convex hull of those permutations that are extensions of a given poset $P$ on the ground set $E$ has aroused considerable interest in the last years, in particular for its application in scheduling (see [8-13]). In this and the next sections, we particularly investigate which properties of these special polytopes are passed to them by the 
more general framework. To be more precise, let $P$ be a poset and $g: \mathscr{I}(P) \rightarrow \mathbb{R}$ a supermodular function on the ideals of $P$. Then, our object of study is the base polytope of the poset $P$ with respect to $g$ which is defined as

$$
B(P, g):=\operatorname{conv}\{x(L): L \in \mathscr{L}(P)\} .
$$

The following results which generalize the respective ones for the permutahedron of a poset (cf., Schulz [14]) can be proved simply by using the (strict) supermodularity of $g$.

Proposition 2. Let $P$ be a poset with $P_{1} \oplus P_{2}$, and let $g: \mathscr{I}(P) \rightarrow \mathbb{B}$ be a supermodular function. Then $B(P, g)$ is the Cartesian product of the polytopes $B\left(P_{1}, g\right)$ and $B\left(P_{2}, g^{\prime}\right)$ where $g^{\prime}(S)=g\left(S \cup P_{1}\right)-g\left(P_{1}\right)$.

A minimal description in terms of linear equations and inequalities for the Cartesian product of given polyhedra can be obtained by the juxtaposition of minimal linear systems of the given polyhedra. Consequently, when studying $B(P, g)$, we may concentrate on posets $P$ that are not series decomposable. We will make use of this property in Section 4. With the help of Proposition 2 it is easy to determine the dimension of $B(P, g)$.

Proposition 3. Let $P$ be a poset with series decomposition $P_{1} \oplus \cdots \oplus P_{q}$ (i.e., $P_{i}$ is not further series decomposable), and let $g: \mathscr{I}(P) \rightarrow \mathbb{R}$ be a strictly supermodular function. Then

$$
x\left(P_{1} \cup \cdots \cup P_{i}\right)=g\left(P_{1} \cup \cdots \cup P_{i}\right) \quad \text { for } i=1,2, \ldots, q
$$

is a minimal linear equation system defining the affine hull of $B(P, g)$. In particular,

$$
\operatorname{dim}(B(P, g))=n-q .
$$

Finally, for strictly supermodular functions $g$, we characterize the facet defining inequalities among those which naturally emerge from the base polytope $B(g)$ of $g$.

Proposition 4. Let $P$ be a poset with series decomposition $P_{1} \oplus \cdots \oplus P_{q}$, and let $I=P_{1} \oplus \cdots \oplus P_{i} \oplus \hat{I}, \quad i \in\{0, \ldots, q-1\}$, be an ideal of $P$. If $\hat{I}_{1} \oplus \cdots \oplus \hat{I}_{r}$ and $\hat{F}_{1} \oplus \cdots \oplus \hat{F}_{s}$ are the series decompositions of $\hat{I}$ and $P_{i+1} \backslash \hat{I}$, respectively, then the face of $B(P, g)$ induced by $x(I) \geqslant g(I)$ is of dimension $n-(q+r+s)+1$.

\section{Base polytopes of series-parallel posets}

For a poset $P$ and a supermodular function $g: \mathscr{I}(P) \rightarrow \mathbb{R}$ compatible with $P$ let $\mathbb{P}(P, g)$ be the polytope defined by the inequalities

$$
|A| x(B)-|B| x(A) \geqslant f(A, B)
$$

for all series-reducible convex sets $(\mathrm{A}, \mathrm{B}), \quad A, B$ series-prime, 


$$
\begin{aligned}
& x(I) \geqslant g(I) \quad \text { for all ideals } I \in \mathscr{I}(P), \\
& x(P)=g(P) .
\end{aligned}
$$

Note that $\mathbb{P}(P, g)$ is not well-defined if $g$ is not compatible with $P$. A subset $A$ of $P$ is called series-prime if $A$ does not allow a series-decomposition. We call the first class of inequalities convex set constraints, and the second class ideal inequalities.

For series parallel posets $P$ and compatible supermodular functions $g$ we will show that $\mathbb{P}(P, g)$ equals $B(P, g)$. In particular, $\mathbb{P}(P, g)$ is integral if $g$ is integral. Our proof follows to a good part the proof given by Arnim et al. [8] for the permutahedron of series-parallel posets. We thereby emphasize the crucial role that the supermodularity of the function $g$ plays. In contrast to the special case of the permutahedron, however, the inclusion $B(P, g) \subseteq \mathbb{P}(P, g)$ is not trivial. To show the validity of the convex set constraints for $B(P, g)$ we need the compatibility of $g$ (see Theorem 8). Justified by Proposition 2, we assume throughout this chapter, when considering a series-parallel poset $P$, that $P$ is series-prime.

The following observation is immediate.

Lemma 5. Let $P$ be a poset and $g: \mathscr{I}(P) \rightarrow \mathbb{B}$ supermodular and compatible. Let $P^{\prime}=P \backslash$ I for some ideal $I$ of $P, g^{\prime}: \mathscr{F}\left(P^{\prime}\right) \rightarrow \mathbb{R}$ with $g^{\prime}(J):=g(I \cup J)-g(I)$ and $f^{\prime}$ the function induced by $g^{\prime}$. Then $g^{\prime}$ is supermodular and compatible with $P^{\prime}$ and $f^{\prime}=f$ on $P \backslash I$.

Given a vector $x \in \mathbb{P}(P, g)$, we call an ideal $I$ tight at $x$ if $x(I)=g(I)$ holds.

Lemma 6. Let $P$ be a poset and $g: \mathscr{I}(P) \rightarrow \mathbb{B}$ supermodular and compatible. Let $x \in \mathbb{P}(P, g)$ and let $I$ be a tight ideal. Then $y=\left(x_{i}: i \in I\right) \in \mathbb{P}(I, g)$ and $z=\left(x_{i}: i \in P \backslash I\right) \in \mathbb{P}\left(P \backslash I, g^{\prime}\right)$ where $g^{\prime}(J)=g(I \cup J)-g(I)$, as above.

Proof. The previous lemma implies that $z$ satisfies the convex set constraints and $y$ satisfies both ideal and convex set constraints. Since $I$ is tight, we also have $y(I)=g(I)$, i.e., $y \in \mathbb{P}(I, g)$. For an ideal $J \subseteq P \backslash I$, we have $z(J)=x(J)=x(I \cup J)$ $-x(I) \geqslant g(I \cup J)-g(I)=g^{\prime}(J)$, i.e., $z$ satisfies the ideal constraints induced by $g^{\prime}$. Finally, $\quad z(P \backslash I)=x(P \backslash I)=x(P)-x(I)=g(P)-g(I)=g^{\prime}(P \backslash I), \quad$ i.e., $\quad z \in \mathbb{P}(P$ $\left.\backslash, g^{\prime}\right)$

Proposition 7. Let $P$ be a series-parallel poset and $g: \mathscr{I}(P) \rightarrow \mathbb{R}$ supermodular and compatible. Then $\mathbb{P}(P, g) \subseteq B(P, g)$ holds.

Proof. (i) We first show by induction that $B(P, g)$ contains $\mathbb{P}(P, g)$ if for any vertex of $\mathbb{P}(P, g)$ there exists a tight proper ideal. For $|P|=1$ the claim obviously holds. Now let $x$ be a vertex of $\mathbb{P}(P, g)$ and $I$ be a tight proper ideal. By Lemma 6 and induction, $y=\left(x_{i}: i \in I\right) \in \mathbb{P}(I, g) \subseteq B(I, g)$ and $z=\left(x_{i}: i \in P \backslash I\right) \in \mathbb{P}\left(P \backslash I, g^{\prime}\right) \subseteq B\left(P \backslash I, g^{\prime}\right)$. Hence, $y$ and $z$ are convex combinations of incidence vectors of linear extensions of $I$ 
and $P \backslash I$, respectively. It follows that $x$ itself is a convex combination of incidence vectors of linear extensions of $P$ and thus contained in $B(P, g)$.

(ii) It remains to show that we can find tight proper ideals. Remember that we assumed that $P=P_{1} \| P_{2}$. Suppose that $x$ is a vertex of $\mathbb{P}(P, g)$ with no tight proper ideal. Then $\epsilon:=\min \{x(I)-g(I): I$ is a proper ideal of $P\}$ is positive. Choose a vector $c \in \mathbb{R}^{E}$, such that $x$ is the unique minimum for $\min \{c z: z \in \mathbb{P}(P, g)\}$. We may assume that $c\left(P_{2}\right)\left|P_{1}\right|-c\left(P_{1}\right)\left|P_{2}\right| \leqslant 0$, otherwise we can renumber $P_{1}$ and $P_{2}$. Now let $y$ be given by

$$
y_{i}= \begin{cases}x_{i}+\epsilon /\left|P_{1}\right| & \text { for } i \in P_{1}, \\ x_{i}-\epsilon /\left|P_{2}\right| & \text { for } i \in P_{2} .\end{cases}
$$

We claim that $y \in \mathbb{P}(P, g)$. Obviously, $y$ lies on the hyperplane $y(P)=g(P)$. Since $P=P_{1} \| P_{2}$, any series-reducible convex set is contained in either $P_{1}$ or $P_{2}$. By using that the convex set constraints are invariant under adding the same constant to every component, it follows for any series-reducible convex set $(A, B)$ that $|A| y(B)-|B| y(A)=|A| x(B)-|B| x(A) \geqslant f(A, B)$. Finally, the ideal inequalities hold for any proper ideal $I \subseteq P$ since

$$
y(I)=\sum_{i \in I \cap P_{1}}\left(x_{i}+\frac{\epsilon}{\left|P_{1}\right|}\right)+\sum_{i \in I \cap P_{2}}\left(x_{i}-\frac{\epsilon}{\left|P_{2}\right|}\right) \geqslant x(I)-\epsilon \geqslant g(I) .
$$

But $c y=c x+\epsilon\left(c\left(P_{1}\right) /\left|P_{1}\right|-c\left(P_{2}\right) /\left|P_{2}\right|\right) \leqslant c x$, contradicting the uniqueness of $x$. Hence, there must exist a tight proper ideal.

Theorem 8. Let $P$ be a series-parallel poset and $g: \mathscr{I}(P) \rightarrow \mathbb{R}$ supermodular and compatible. Then $\mathbb{P}(P, g)$ coincides with $B(P, g)$.

Proof. Because of Proposition 7, it is sufficient to show $B(P, g) \subseteq \mathbb{P}(P, g)$. Let $x=x(L)$ be the incidence vector of a linear extension of $P$. Obviously, $x$ satisfies $x(P)=g(P)$ and the ideal constraints.

Now, let $(A, B)$ be a series-reducible convex set with $A$ and $B$ series-prime. Let $L=e_{1} \ldots e_{n}, j=\max \left\{i: e_{i} \in A\right\} \quad$ and $J=\left\{e_{1}, \ldots, e_{j}\right\}$. Then $x_{J} \in B(J, g)$ and $x_{P \backslash J} \in B\left(P \backslash J, g^{\prime}\right)$. In particular, $x(B) \geqslant g^{\prime}(B)=g(J \cup B)-g(J)$ holds if $B$ is an ideal in $P \backslash J$. If $J \backslash A$ is an ideal of $J$, too, then $x(A)=x(J)-x(J \backslash A) \leqslant g(J)-g(J \backslash A)$. Using $I=J \backslash A$, the desired convex set inequality $|A| x(B)-|B| x(A) \geqslant$ $|A|[g(I \cup A \cup B)-g(I \cup A)]-|B|[g(I \cup A)-g(I)]$ would follow. It remains to show that we can force $J \backslash A$ and $B$ to be ideals in $J$ and $P \backslash J$, respectively.

Suppose $J \cup B$ is not an ideal of $P$. Then there exists a first element $e_{i}=c \in P \backslash(J \cup B)$ in $L$ with $c<b$ for some $b \in B$. From $i>j$ and the convexity of $A \cup B$ we conclude $c \| A$. Since $P$ is series-parallel and $B$ is series-prime, $c<B$ follows. Let $L^{\prime}$ be the linear extension arising from $L$ by exchanging $e_{i}$ and $e_{j}$ with incidence vector $x^{\prime}$. The supermodularity of $g$ implies $x^{\prime}(A) \geqslant x(A)$, whereas $x^{\prime}(B)=x(B)$ remains unchanged. We continue the exchange operations until $J \cup B$ is an ideal of $P$. 
Suppose $I=J \backslash A$ is not an ideal of $P$. Then there exists elements $a \in A$ and $y \in I$ with $a<y$. Since $e_{j}=: a^{\prime}$ belongs to $A$, we know that $y<a^{\prime}$ or $y \| a^{\prime}$. Because $A$ is convex, $y$ must be parallel to $a^{\prime}$. We again distinguish two cases.

(a) $a \| a^{\prime}$. Let $b \in B$ be arbitrary. Then $a, b, a^{\prime}, y$ induce an $N$, which contradicts the fact that $P$ is series-parallel.

(b) $a<a^{\prime}$. Because $A$ is series-prime and $P$ is series-parallel, there must be an element $a^{\prime \prime} \|\left\{a, a^{\prime}\right\}$. If $a^{\prime \prime} \| y$, then $a, b, a^{\prime \prime}, y$ induce an $N$. If $a^{\prime \prime}<y$, then $a^{\prime \prime}, b, a^{\prime}, y$ induce an $N$. Both possibilities contradict that $P$ is series-parallel. Since $A$ is convex, $y$ cannot be a predecessor of $a^{\prime \prime}$.

The characterization of $B(P, g)$ given in Theorem 8 generalizes similar characterizations of v. Arnim et al. [8] for the permutahedron, and of Queyranne and Wang [10] for a related scheduling polyhedron.

\section{Optimization}

Let $P$ be a poset, $g: \mathscr{I}(P) \rightarrow \mathbb{R}$ a supermodular function and $c \in \mathbb{R}^{P}$. Consider the linear programming problem

$$
\operatorname{opt}(P, g, c):=\min \{c x: x \in B(P, g)\} \text {. }
$$

Since $x(P)=g(P)$, we may assume that $c$ is nonnegative.

Call an ideal $I \rho$-maximal if $\rho(I)=c(I) /|I| \geqslant c(J) /|J|=\rho(J)$ for all ideals $J \subseteq P$. The algorithm we propose to solve opt $(P, g, c)$ for series-parallel posets and supermodular and compatible functions $g$ is a generalization of Sidney's algorithm [1] for minimizing the weighted sum of completion times in a one-machine scheduling environment. It starts with some $\rho$-maximal ideal $I$ and solves the problem recursively on $I$ and $P \backslash I$. In general, $P$ itself may be the only $\rho$-maximal ideal and the approach does not work. However, if $P$ decomposes into two parallel components, there is always a $\rho$-maximal ideal that is contained in one of the components. This is implied by the following observation which holds for all $x, y \geqslant 0$ and $X, Y \in \mathbb{R}$ :

$$
(X+Y) /(x+y) \leqslant X / x \Longleftrightarrow Y / y \leqslant X / x \text {. }
$$

Lemma 9. Let $P$ be a poset, $g: \mathscr{I}(P) \rightarrow \mathbb{R}$ supermodular and $c \in \mathbb{R}^{P}$. Let $L=L_{I} L_{P \backslash I}$ be an optimal linear extension for $\operatorname{opt}(P, g, c)$. Then any linear extension $L^{\prime}=L_{I}^{\prime} L_{P \backslash I}^{\prime}$ starting with $I$ is optimal for opt $(P, g, c)$ if and only if $L_{I}^{\prime}$ is optimal for opt $\left(I, g, c_{I}\right)$ and $L_{P \backslash I}^{\prime}$ is optimal for $\operatorname{opt}\left(P \backslash I, g^{\prime}, c_{P \backslash I}\right)$, where $g^{\prime}(J)=g(I \cup J)-g(I)$, as above.

Proof. By definition of $B(P, g)$ and $g^{\prime}$ we have $\operatorname{opt}(P, g, c) \leqslant \operatorname{opt}\left(I, g, c_{I}\right)+$ $\operatorname{opt}\left(P \backslash I, g^{\prime}, c_{P \backslash I}\right)$. Since $L$ is optimal for opt $(P, g, c)$, equality follows.

Lemma 10. Let $P$ be a poset and $g: \mathscr{I}(P) \rightarrow \mathbb{R}$ be supermodular and compatible. Let $L=L_{I} a_{1} \ldots a_{r} b_{1} \ldots b_{s} L_{J}$ be a linear extension of $P$ with incidence vector $x$ containing $a$ 
series-reducible convex set $(A, B)=\left(\left\{a_{1}, \ldots, a_{r}\right\},\left\{b_{1}, \ldots, b_{s}\right\}\right)$. Then, using $A_{j}:=\left\{a_{j}, \ldots, a_{r}\right\}$ and $B_{j}:=\left\{b_{1}, \ldots, b_{j}\right\}$, we obtain

$$
\begin{aligned}
x\left(b_{j}\right)= & x\left(a_{1}\right)+f\left(A, b_{1}\right)-f\left(A_{2}, b_{1}\right)-f\left(A_{r}, b_{1}\right)+f\left(A_{r}, B_{j}\right) \\
& +f\left(A_{r}, B_{j-1}\right) \text { for } j=1, \ldots, s
\end{aligned}
$$

and

$$
\begin{gathered}
x\left(a_{j}\right)=x\left(a_{1}\right)+f\left(A, b_{1}\right)-f\left(A_{2}, b_{1}\right)-f\left(A_{j}, b_{1}\right)+f\left(A_{j+1}, b_{1}\right) \\
\text { for } j=1, \ldots, r .
\end{gathered}
$$

Proof. The convex set constraints for $A_{j} \oplus\left\{b_{1}\right\}, 1 \leqslant j \leqslant r$, and $\left\{a_{r}\right\} \oplus B_{j}, 1 \leqslant j \leqslant s$, are tight at $x(L)$.

By induction on $j$ we first show $x\left(b_{j}\right)=x\left(a_{r}\right)+f\left(a_{r}, B_{j}\right)-f\left(a_{r}, B_{j-1}\right)$. For $j=1$, the tight convex set constraint for $\left\{a_{r}\right\} \oplus\left\{b_{1}\right\}$ gives $x\left(b_{1}\right)-x\left(a_{r}\right)=f\left(a_{r}, b_{1}\right)=f\left(a_{r}, B_{1}\right)-f\left(a_{r}, \emptyset\right)$. In the induction step we use the tight convex set constraint for $\left\{a_{r}\right\} \oplus B_{j}$. With $\sum_{i=1}^{j} x\left(b_{i}\right)-j x\left(a_{r}\right)=f\left(a_{r}, B_{j}\right)$ we get

$$
\begin{aligned}
x\left(b_{j}\right)= & f\left(a_{r}, B_{j}\right)+j x\left(a_{r}\right)-\sum_{i=1}^{j-1} x\left(b_{i}\right)=f\left(a_{r}, B_{j}\right)+j x\left(a_{r}\right)-(j-1) x\left(a_{r}\right) \\
& +\sum_{i=1}^{j-1}\left(-f\left(a_{r}, B_{i}\right)+f\left(a_{r}, B_{i-1}\right)\right)=f\left(a_{r}, B_{j}\right)+x\left(a_{r}\right)-f\left(a_{r}, B_{j-1}\right) .
\end{aligned}
$$

By symmetry, $x\left(a_{j}\right)=x\left(b_{1}\right)-f\left(A_{j}, b_{1}\right)+f\left(A_{j+1}, b_{1}\right)$ holds. Using $x\left(a_{1}\right)=x\left(b_{1}\right)-$ $f\left(A, b_{1}\right)+f\left(A_{2}, b_{1}\right)$ to substitute for $x\left(b_{1}\right)$ in the equation for $x\left(a_{j}\right)$, we obtain $x\left(a_{j}\right)=x\left(a_{1}\right)+f\left(A, b_{1}\right)-f\left(A_{2}, b_{1}\right)-f\left(A_{j}, b_{1}\right)+f\left(A_{j+1}, b_{1}\right)$. Using this equation to substitute for $x\left(a_{r}\right)$ in the equation for $x\left(b_{j}\right)$, we finally obtain $x\left(b_{j}\right)=x\left(a_{1}\right)+f\left(A, b_{1}\right)-f\left(A_{2}, b_{1}\right)-f\left(A_{r}, b_{1}\right)+f\left(A_{r}, B_{j}\right)+f\left(A_{r}, B_{j-1}\right)$.

Corollary 11. Let $P$ and $g$ be as in Lemma 10 , let $L=L_{I} e_{1} \ldots e_{k} L_{J}$ be a linear extension of $P$ with incidence vector $x$ and let $\left\{e_{1}, \ldots, e_{k}\right\}$ be a series-reducible convex set of $P$. Then

$$
x\left(e_{i}\right)=x\left(e_{1}\right)+s_{i},
$$

and the values of $s_{i}$ are independent of $L$ on $P \backslash\left\{e_{1}, \ldots, e_{k}\right\}$.

Proposition 12. Let $P$ be a poset, $g: \mathscr{I}(P) \rightarrow \mathbb{R}$ strictly supermodular and compatible and $c \in \mathbb{R}^{P}$. Let $L=L_{I} e_{1} \ldots e_{k} f_{1} \ldots f_{l} L_{J}$ and $L^{\prime}=L_{I} f_{1} \ldots f_{l} e_{1} \ldots e_{k} L_{J}$ be two linear extensions of $P$ with incidence vectors $x$ and $y$ and series-reducible convex sets $E=\left\{e_{1}, \ldots, e_{k}\right\}$ and $F=\left\{f_{1}, \ldots, f_{l}\right\}$. Then $c x \leqslant c y$ holds if and only if $\rho(E) \geqslant \rho(F)$ holds.

Proof. Using Corollary 11, we can write 


$$
\begin{array}{ll}
x\left(e_{i}\right)=x\left(e_{1}\right)+t_{i}, & x\left(f_{j}\right)=x\left(f_{1}\right)+s_{j}, \\
y\left(e_{i}\right)=y\left(e_{1}\right)+t_{i}, & y\left(f_{j}\right)=y\left(f_{1}\right)+s_{j}
\end{array}
$$

for all $1 \leqslant i \leqslant k, 1 \leqslant j \leqslant l$. Then $c x \leqslant c y$ is equivalent to

$$
\begin{aligned}
& \sum_{i=1}^{k} c_{e_{i}} x\left(e_{i}\right)+\sum_{j=1}^{l} c_{f_{j}} x\left(f_{j}\right) \leqslant \sum_{i=1}^{k} c_{e_{i}} y\left(e_{i}\right)+\sum_{j=1}^{l} c_{f_{j}} y\left(f_{j}\right) \\
& \Longleftrightarrow c(E) x\left(e_{1}\right)+c(F) x\left(f_{1}\right) \leqslant c(E) y\left(e_{1}\right)+c(F) y\left(f_{1}\right) \\
& \Longleftrightarrow c(E)\left[g\left(I \cup e_{1}\right)-g(I)-g\left(I \cup F \cup e_{1}\right)+g(I \cup F)\right] \\
& \leqslant c(F)\left[g\left(I \cup f_{1}\right)-g(I)-g\left(I \cup E \cup f_{1}\right)+g(I \cup E)\right] .
\end{aligned}
$$

We have $g(I \cup E)-g(I)=\sum_{i=1}^{k} x\left(e_{i}\right)=k x\left(e_{1}\right)+\sum_{i=1}^{k} t_{i}=k g^{\prime}\left(e_{1}\right)+\sum_{i=1}^{k} t_{i}, \quad$ and $g(I \cup F)-g(I)=\lg ^{\prime}\left(f_{1}\right)+\sum_{j=1}^{l} s_{j}$. Using the linear extension $L_{I} e_{1} f_{1} \ldots f_{l} e_{2} \ldots e_{k} L_{J}$ it follows that $g\left(I \cup F \cup e_{1}\right)-g\left(I \cup e_{1}\right)=l\left(g^{\prime}\left(\left\{e_{1}, f_{1}\right\}\right)-g^{\prime}\left(e_{1}\right)\right)+\sum_{j=1}^{l} s_{j}$, and similarly $g\left(I \cup E \cup f_{1}\right)-g\left(I \cup f_{1}\right)=k\left(g^{\prime}\left(\left\{e_{1}, f_{1}\right\}\right)-g^{\prime}\left(f_{1}\right)\right)+\sum_{i=1}^{l} t_{i}$. Substituted into the last inequality above we get

$$
c(E)\left[l\left(g^{\prime}\left(f_{1}\right)+g^{\prime}\left(e_{1}\right)-g^{\prime}\left(\left\{f_{1}, e_{1}\right\}\right)\right)\right] \leqslant c(F)\left[k\left(g^{\prime}\left(f_{1}\right)+g^{\prime}\left(e_{1}\right)-g^{\prime}\left(\left\{f_{1}, e_{1}\right\}\right)\right)\right] .
$$

Since $g^{\prime}\left(\left\{e_{1}, f_{1}\right\}\right)-g^{\prime}\left(e_{1}\right)-g^{\prime}\left(f_{1}\right)>0$ ( $g$ is strictly supermodular) the last inequality holds if and only if $c(E) /|E| \geqslant c(F) /|F|$ holds.

Again, let $L=e_{1} \ldots e_{n}$ be a linear extension of $P$. We call an interval $I=e_{i} e_{i+1} \ldots e_{k}$ of $L$ series-reducible, if $\left\{e_{i}, e_{i+1}, \ldots, e_{k}\right\}$ is a series-reducible convex set of $P$. The interval $I$ is called maximal series-reducible (in $L$ ), if no larger series-reducible interval $J \nsupseteq I$ in $L$ exists.

Proposition 13. Let $P$ be a series parallel poset. Let $L$ be a linear extension with (in this order, from the beginning of $L$ to its end) maximal series-reducible intervals $E_{i}, 1 \leqslant i \leqslant s$, i.e., $E_{i}$ is a singleton or $E_{i}=A_{i} \oplus B_{i}$ with nonempty subsets $A_{i}$ and $B_{i}$. Then

$$
E_{i} \| E_{i+1} \quad \text { for all } 1 \leqslant i<s .
$$

Proof. We proceed by induction on $n=|P|$. If $n=1$ or $P=P_{1} \oplus P_{2}$, we must have $s=1$ and we are done. Now let $P=P_{1} \| P_{2}$. Suppose the first element $e_{1}$ of $L$ belongs to $P_{1}$. Let $e_{i}$ be the first element in $L$ out of $P_{2}$. By the induction hypothesis, the claim is valid for the linear extensions $e_{1} \ldots e_{i-1}$ and $e_{i} \ldots e_{n}$. The last maximal seriesreducible interval in $e_{1} \ldots e_{i-1}$ is a subset of $P_{1}$, and the first maximal series-reducible interval in $e_{i} \ldots e_{n}$ is a subset of $P_{2}$, so they are parallel to each other, too.

Corollary 14. Let $P$ be a series-parallel poset, $g: \mathscr{I}(P) \rightarrow \mathbb{R}$ strictly supermodular and compatible, and $c \in \mathbb{R}^{P}$. Let $L=L_{I} e_{1} \ldots e_{k} f_{1} \ldots f_{l} L_{J}$ be an optimal linear extension for $\operatorname{opt}(P, g, c)$ and $L^{\prime}=L_{I} f_{1} \ldots f_{l} e_{1} \ldots e_{k} L_{J}$ be a linear extension of $P$, too. Let $E=\left\{e_{1}, \ldots, e_{k}\right\}$ and $F=\left\{f_{1}, \ldots, f_{k}\right\}$. Then, $L^{\prime}$ is optimal for $\operatorname{opt}(P, g, c)$ if and only if $\rho(F) \geqslant \rho(E)$. 
Proof. Let $E=E_{1}\|\cdots\| E_{s}$ and $F=F_{1}\|\cdots\| F_{t}$ be the decomposition of $E$ and $F$ in maximal series-reducible intervals in $L$, respectively. Since $L$ is optimal and $L^{\prime}$ is valid, Propositions 13 and 12 imply

$$
\rho\left(E_{1}\right) \geqslant \rho\left(E_{2}\right) \geqslant \cdots \geqslant \rho\left(E_{s}\right) \geqslant \rho\left(F_{1}\right) \geqslant \rho\left(F_{2}\right) \geqslant \cdots \geqslant \rho\left(F_{t}\right) .
$$

Observe that $L$ can be transformed into $L^{\prime}$ by swapping $E_{i}$ with $F_{j}$, $j=1, \ldots, t, i=s, \ldots, 1$. By Proposition 12 , every swap does not decrease the objective function value.

Let $L^{\prime}$ be optimal, too. Suppose $\rho(F)<\rho(E)$. Then $\rho\left(E_{i_{0}}\right)>\rho\left(F_{j_{0}}\right)$ for at least one pair $\left(i_{0}, j_{0}\right)$. Swapping $E_{i_{0}}$ with $F_{j_{0}}$ increases the objective value, any other swap operation does not decrease the objective value. This contradicts the optimality of $L^{\prime}$.

In the other direction, $\rho(F) \geqslant \rho(E)$ and the inequalities (3) and (7) imply $\rho(F)=\rho(E)$. Consequently, equality in Eq. (7) follows. Now, by Proposition 12 every swap operation keeps the objective function value constant. Hence $L^{\prime}$ is optimal, too.

This proves the "adjacent string interchange property" (see, e.g., [15]) for optimal linear extensions.

Like Sidney, we call an ideal $I \rho^{\star}$-maximal if it is $\rho$-maximal and does not contain a smaller ideal which is $\rho$-maximal, too.

Theorem 15. Let $P$ be a series-parallel poset, $g: \mathscr{I}(P) \rightarrow \mathbb{R}$ strictly supermodular and compatible and $c \in \mathbb{R}^{P}$. Let $L$ be an optimal linear extension for opt $(P, g, c)$. Then $L$ starts with a $\rho^{\star}$-maximal ideal $I$.

Proof. We proceed by induction on $n=|P|$. The case $n=1$ is trivial. By Lemma 9, it is sufficient to consider the case $P=P_{1} \| P_{2}$ in the induction step. Let $L_{1}$ be the $\rho^{\star}-$ maximal initial ideal of $L=e_{1} \ldots e_{n}$, i.e., among all initial ideals of $L$ the smallest one with maximal $\rho$-value. To be more precise, let $j_{0}:=\min \{j: \forall 1 \leqslant k \leqslant$ $\left.n: \rho\left(\left\{e_{1}, \ldots, e_{j}\right\}\right) \geqslant \rho\left(\left\{e_{1}, \ldots, e_{k}\right\}\right)\right\}$. Then $L_{1}=e_{1}, \ldots, e_{j_{0}}$ and we denote by $I_{1}$ the underlying set, i.e., $I_{1}=\left\{e: e \in L_{1}\right\}$. By Propositions 13 and $12, L_{1}$ is a singleton or series-reducible. Hence $I_{1}$ is entirely included in $P_{1}$ or in $P_{2}$. W.l.o.g., we assume that $I_{1} \subseteq P_{1}$. By Lemma $9, L_{1}$ is optimal for $\operatorname{opt}\left(I_{1}, g, c_{I_{1}}\right)$ and $L \backslash L_{1}$ is optimal for $\operatorname{opt}\left(P \backslash I_{1}, g^{\prime}, c_{P \backslash I_{1}}\right)$. We claim that $I_{1}$ is a $\rho^{\star}$-maximal ideal in $P$.

By the induction hypothesis, $L_{1}$ starts with a $\rho^{\star}$-maximal ideal of $I_{1}$. The choice of $I_{1}$ assures this is $I_{1}$. Hence, $I_{1}$ does not include a smaller ideal with the same or a bigger $\rho$-value.

Again by the induction hypothesis, $L \backslash L_{1}$ starts with a $\rho^{\star}$-maximal ideal $J \subseteq P \backslash I_{1}$. Now, if $\rho\left(I_{1}\right)<\rho(J)$ then equivalence (3) implies that $\rho\left(I_{1}\right)<\rho\left(I_{1} \cup J\right)$. This contradicts the choice of $L_{1}$. Consequently, the $\rho$-value of any ideal in $P \backslash I_{1}$ is at most $\rho\left(I_{1}\right)$.

It remains to consider any ideal $K$ of $P$ such that $K \cap I_{1} \neq \emptyset$ and $K \cap\left(P \backslash I_{1}\right) \neq \emptyset$. However, again by use of Eq. (3), $\rho\left(K \cap I_{1}\right) \leqslant \rho\left(I_{1}\right)$ and $\rho\left(K \cap\left(P \backslash I_{1}\right)\right) \leqslant \rho(J)$ imply $\rho(K) \leqslant \rho\left(I_{1}\right)$. 
The next two lemmas are direct extensions of their counterparts in Sidney's theory [1]. Lemma 17 corresponds to Sidney's "Main Decomposition Theorem".

Lemma 16. Let $P$ be a poset and $c \in \mathbb{R}^{P}$. Then, the $\rho^{\star}$-maximal ideals of $P$ are pairwise disjoint.

Proof. Let $I$ and $J$ be two distinct $\rho^{\star}$-maximal ideals. Hence, $I \nsubseteq J$ and vice versa. Suppose that $K=I \cap J$ is nonempty. Then, $K$ is an ideal with $\rho(K)<\rho(I)=\rho(J)$. In this case, using equivalence (3), $\rho(I \backslash K)>\rho(K)$ and $\rho(I \backslash K)>\rho(I)$ follow. Again using equivalence (3), $\rho(J \cup(I \backslash K))>\rho(I)$ would follow which contradicts the $\rho$ maximality of $I$.

Lemma 17. Let $P$ be a poset and $c \in \mathbb{R}^{P}$. Let $S_{1} S_{2} \ldots S_{a}$ and $T_{1} T_{2} \ldots T_{b}$ be two sequences of $\rho^{\star}$-maximal ideals (i.e., $S_{j}$ is $\rho^{\star}$-maximal in $P \backslash \cup_{i=1}^{j-1} S_{i}$ for $1 \leqslant j \leqslant a$, and $T_{j}$ is $\rho^{\star}$-maximal in $P \backslash \cup_{i=1}^{j-1} T_{i}$ for $\left.1 \leqslant j \leqslant b\right)$. Then $a=b$, and there exists $a$ permutation $\pi$ such that $S_{i}=T_{\pi(i)}$ for all $i$.

Proof. We proceed by induction on $n=|P|$. The induction start is trivial. If $S_{1}=T_{1}$ holds in the induction step, we are finished by the induction hypothesis. Hence, assume $S_{1} \neq T_{1}$. From Lemma 16 we know that $S_{1} \cap T_{1}=\emptyset$. Notice that $T_{1}$ is $\rho$ maximal in $P \backslash S_{1}$, too. By the induction hypothesis for $P \backslash S_{1}$ we can conclude that $T_{1} \in\left\{S_{2}, \ldots, S_{a}\right\}$. Now the exchange of $S_{i}:=T_{1}$ and $S_{1}$ in the sequence $S_{1} S_{2} \ldots S_{a}$ leads us back to the previous case.

Corollary 18. Let $P$ be a poset and $c \in \mathbb{R}^{P}$. Every $\rho$-maximal ideal is the disjoint union of $\rho^{\star}$-maximal ideals.

Theorem 19. Let $P$ be a series-parallel poset, $g: \mathscr{I}(P) \rightarrow \mathbb{R}$ strictly supermodular and compatible, and $c \in \mathbb{R}^{P}$. Let $S$ be a $\rho^{\star}$-maximal ideal. Then there exists an optimal linear extension of $P$ for $\operatorname{opt}(P, g, c)$ starting with $S$.

Proof. Let $L=S_{1} S_{2} \ldots S_{a}$ with $S=S_{1}$ be a linear extension of $P$ such that, for each $j=1, \ldots, a$, the set $S_{j}$ is a $\rho^{\star}$-maximal in $P \backslash \cup_{i=1}^{j-1} S_{i}$. By Theorem 15 there exists a linear extension $L^{\prime}$ of $P$ optimal for $\operatorname{opt}(P, g, c)$ which starts with a $\rho^{\star}$-maximal ideal $T_{1}$ of $P$. By Lemma 9, $L^{\prime} \backslash T_{1}$ is optimal for $P \backslash T_{1}$. By induction, $L^{\prime}=T_{1} T_{2} \ldots T_{b}$ where $T_{j}$ is $\rho^{\star}$-maximal in $P \backslash \cup_{i=1}^{j-1} T_{i}$. Because of Lemma 17 there must be a $k$ with $T_{k}=S_{1}=S$. In the case $k=1$ we are done. Otherwise, let $L^{\prime}=T_{1} \ldots T_{k-1} S_{1} T_{k+1} \ldots T_{b}=E_{1} E_{2} \ldots E_{l} S_{1} T_{k+1} \ldots T_{b}$, where the $E_{i}$ are maximal series-reducible intervals in $L^{\prime}$. From Propositions 13 and 12, $\rho\left(E_{i}\right) \geqslant \rho\left(E_{i+1}\right)$ for all $i$. Since $T_{1}$ is $\rho^{\star}$-maximal, $\rho\left(S_{1}\right)=\rho\left(T_{1}\right) \geqslant \rho\left(E_{1}\right)$ holds. Successive swaps of $S_{1}$ with $E_{i}(i=l, l-1, \ldots, 1)$ do not alter the objective value by Proposition 12 . Consequently, $L^{\prime \prime}=S_{1} T_{1} \ldots T_{k-1} T_{k+1} \ldots T_{b}$ is an optimal linear extension starting with $S$. 
Although Theorem 19 is apparently restricted to strictly supermodular functions, it already provides most of the ingredients for the general case.

Corollary 20. Let $P$ be a series-parallel poset, $g: \mathscr{I}(P) \rightarrow \mathbb{R}$ supermodular and compatible, and $c \in \mathbb{R}^{P}$. Let $S$ be a $\rho^{\star}$-maximal ideal. Then there exists an optimal linear extension of $P$ for $\operatorname{opt}(P, g, c)$ starting with $S$.

Proof. We define $h_{\epsilon}: \mathscr{I}(P) \rightarrow \mathbb{R}$ by $h_{\epsilon}(I):=\epsilon \sum_{i=1}^{|I|} i$ for all $\epsilon>0$. The function $h_{\epsilon}$ is strictly supermodular and compatible. Hence, $g+h_{\epsilon}$ is strictly supermodular and compatible, too. Let $K=\max \left\{c x: x \in B\left(P, h_{1}\right)\right\}>0$ (recall that we may assume that $c>0$ ) and let $d \geqslant 0$ be a lower bound for the difference of the second best objective function value of a linear extension with $\operatorname{opt}(P, g, c)$. If all linear extensions are optimal, there is nothing to show. Now we choose a positive $\epsilon<d / K$. By Theorem 19 there exists an optimal linear extension $L$ for opt $\left(P, g+h_{\epsilon}, c\right)$ starting with $S$. Since

$$
\begin{aligned}
& \operatorname{opt}(P, g, c)+\operatorname{opt}\left(P, h_{\epsilon}, c\right) \leqslant \operatorname{opt}\left(P, g+h_{\epsilon}, c\right)<\operatorname{opt}(P, g, c)+K d / K \\
& \quad \leqslant \min \{c x: x \in \operatorname{vert}(B(P, g)), c x>\operatorname{opt}(P, g, c)\},
\end{aligned}
$$

$L$ must be optimal for opt $(P, g, c)$, too.

As in Sidney's paper the proof above shows that in general we can start with an arbitrary $\rho^{*}$-maximal ideal to construct an optimal linear extension. Hence, the algorithm of Lawler [16] for optimizing over series-parallel ordered sets can be used here, too.

Theorem 21. Let $P$ be a series parallel poset and $g: \mathscr{I}(P) \rightarrow \mathbb{R}$ be supermodular and compatible. Let $c \in \mathbb{Q}^{P}$. The optimization problem opt $(P, g, c)$ can be solved in $\mathrm{O}(n \log n)$ time.

In the case of an antichain $P$, the algorithm reduces to the greedy algorithm with the initial sorting phase done by mergesort.

\section{Remarks and open questions}

Base polytopes of series-parallel posets are a common generalization of base polytopes over sets (cf., Fujishige [3]) and permutahedra of series-parallel posets which are defined as follows. With any permutation $\pi$ of an $n$-element set $E=\{1, \ldots, n\}$ we associate a permutation vector via $x(\pi):=(\pi(1), \ldots, \pi(n)) \in \mathbb{R}^{E}$. For a partially ordered set $P=\left(E,<_{P}\right)$, we consider only those permutations which are linear extensions of the poset and define the permutahedron

$$
\operatorname{perm}(P)=\operatorname{conv}\{x(\pi): \pi \text { is a linear extension of } P\} .
$$

In $[8,10]$ it is shown that the permutahedron of a series-parallel poset is given by the linear inequalities 


$$
\begin{aligned}
& |A| x(B)-|B| x(A) \geqslant \frac{1}{2}|A||B|(|A|+|B|) \\
& \quad \text { for all series-reducible convex sets }(A, B), \\
& x(I) \geqslant \frac{1}{2}|I|(|I|+1) \quad \text { for all ideals } I \in \mathscr{I}(P), \\
& x(P)=\frac{1}{2}|P|(|P|+1) .
\end{aligned}
$$

It is easily seen that $g(I)=\frac{1}{2}|I|(|I|+1)$ is strictly supermodular and compatible and $f(A, B)=\frac{1}{2}|A||B|(|A|+|B|)$ is the function induced by $g$. Queyranne and Wang [10] (see also [12]) extend this characterization to the generalized permutahedron which corresponds to the weighted case discussed below.

Queyranne and Schulz [13] show that the problem of scheduling jobs with unit execution times and compatible release dates on $m$ machines with nonstationary speeds may be formulated in terms of optimizing linear functions over contra-polymatroids. For example, for the case of zero release dates, let $P$ be an antichain of $n$ jobs, and suppose there are $m$ machines $i=1, \ldots, m$ with processing rates $\sigma_{i}(\tau) \geqslant 0$. Define $t(i, 0):=0$ and $t(i, k):=\min \left\{t: \int_{t(i, k-1)}^{t} \sigma_{i}(\tau) \mathrm{d} \tau=1\right\}$ as the earliest completion time of the $k$ th job on machine $i$. For $A \subseteq P$, let $\phi(A)$ be the sum of the $|A|$ smallest elements in the multiset $\{t(i, k): 1 \leqslant i \leqslant m, 1 \leqslant k \leqslant n\}$. Then $\phi$ is supermodular, and the completion time vectors of all schedules (with minimum makespan) is $\left\{x \in \mathbb{R}^{P}: x(A) \geqslant \phi(A)\right.$ for all $\left.A \subseteq P, x(P)=\phi(P)\right\}$. By using the results of Section 3, we can immediately extend this description to weak orders. (A poset $P$ is a weak order, if it is the series-composition $A_{1} \oplus \cdots \oplus A_{s}$ of a family of antichains $A_{i}$.) In this case, the compatibility requirement 2 is trivial because $I=I(B) \backslash(A \cup B)$ is unique, and the supermodularity of $\phi(A):=\min \{x(A): x$ is a completion time vector of minimum makespan schedule $\}$ follows from the supermodularity of $\phi$ on an antichain.

We have used the cardinality function in the convex set constraints to simplify the presentation. The arguments carry over to any positive weight function $w: E \rightarrow \mathbb{R}_{+}$. For a linear extension $L=e_{1} \ldots e_{n}$ of $P$, let the weighted incidence vector $x$ of $L$ be the vector with components $x_{e_{i}}=1 / w_{c_{i}}\left(g\left(\left\{e_{1}, \ldots, e_{i}\right\}\right)-g\left(\left\{e_{1}, \ldots, e_{i-1}\right\}\right)\right)$ for $1 \leqslant i \leqslant n$. Let $x_{w}(I)=\sum_{i \in I} w_{i} x_{i}$ and call a supermodular function $g: \mathscr{I}(P) \rightarrow \mathbb{R}$ w-compatible (on $P$ ) if for all series-reducible convex sets $(A, B)$ and any $I \in \mathscr{I}(P), I \subseteq E \backslash(A \cup B)$ such that $I \cup A \in \mathscr{I}(P)$ and $I \cup A \cup B \in \mathscr{I}(P)$, and the term

$$
w(A)[g(I \cup A \cup B)-g(I \cup A)]-w(B)[g(I \cup A)-g(I)]
$$

is a constant independent of $I$. Consider the polytope $\mathbb{P}_{w}(P, g)$ defined by the inequalities

$$
\begin{aligned}
& w(A) x_{w}(B)-w(B) x_{w}(A) \geqslant f(A, B) \\
& \text { for all series-reducible convex sets }(A, B), A, B \text { series-prime, } \\
& x_{w}(I) \geqslant g(I) \text { for all ideals } I \in \mathscr{I}(P), \\
& x_{w}(P)=g(P) .
\end{aligned}
$$

It is a technical exercise to derive the following corollary. 
Corollary 22. Let $P$ be a series-parallel poset $P$ and $g: \mathscr{F}(P) \rightarrow \mathbb{R}$ be a supermodular and $w$-compatible function. Then a vector $x$ is a vertex of $\mathbb{P}_{w}(P, g)$ if and only if it is the weighted incidence vector of a linear extension.

We close with two open questions. First, the inequalities $|A| x(B)-|B| x(A) \geqslant f(A, B)$ closely resemble the defining system of pseudomatroids (cf. [17]). Second, Faigle and Kern [18] have introduced another type of greedy algorithm on posets, also generalizing the polymatroidal procedure. In both cases, it is not clear how these approaches relate to the base polytope of a poset.

\section{References}

[1] J.B. Sidney, Decomposition algorithms for single-machine sequencing with precedence relations and deferral costs, Operations Research 23 (1975) 283-298.

[2] W.E. Smith, Various optimizers for single-stage production, Naval Research and Logistics Quarterly 3 (1956) 59-66.

[3] S. Fujishige, Submodular Functions and Optimization, Annals of Discrete Mathematics, vol. 47, North-Holland, Amsterdam, 1991.

[4] D. Seinsche, On a property of the class of n-colourable graphs, Journal of Combinatorial Theory, Series B 16 (1974) 191-193.

[5] E. Balas, A linear characterization of permutation vectors, Management Science Research Report 364, Carnegie Mellon University, Pittsburgh, 1975.

[6] P. Gaiha, S.K. Gupta, Adjacent vertices on a permutohedron, SIAM Journal of Applied Mathematics 32 (1977) 323-327.

[7] A. Vince, A rearrangement inequality and the permutahedron, The American Mathematical Monthly 97 (1990) 319-323.

[8] A.v. Arnim, U. Faigle, R. Schrader, The permutahedron of series-parallel posets, Discrete Applied Mathematics 28 (1990) 3-9.

[9] A.S. Schulz, The permutahedron of series-parallel posets, Discrete Applied Mathematics 57 (1995) $85-90$.

[10] M. Queyranne, Y. Wang, Single-machine scheduling polyhedra with precedence constraints, Mathematics of Operations Research 16 (1991) 1-29.

[11] A.v. Arnim, R. Schrader, Y. Wang, The permutahedron of N-sparse posets, Mathematical Programming 75 (1996) 1-18.

[12] A.v. Arnim, A.S. Schulz, Facets of the generalized permutahedron of a poset, Discrete Applied Mathematics 72 (1997) 179-192.

[13] M. Queyranne, A.S. Schulz, Scheduling jobs with compatible release dates on parallel machines with nonstationary speeds, in: E. Balas, J. Clausen (Eds.), Integer Programming and Combinatorial Optimization, Lecture Notes in Computer Science 920, Springer, Berlin, 1995, pp. 307-320.

[14] A.S. Schulz, Polytopes and Scheduling, Ph.D. Thesis, Department of Mathematics, Technical University of Berlin, 1996.

[15] E.L. Lawler, J.K. Lenstra, A.H.G. Rinnooy Kan, D.B. Shmoys, Sequencing and scheduling: Algorithms and complexity, in: S.C. Graves, A.H.G. Rinnooy Kan, P.H. Zipkin (Eds.), Logistics of Production and Inventory, Handbooks in Operations Research and Management Science, vol. 4, North-Holland, Amsterdam, 1993, pp. 445-522.

[16] E.L. Lawler, Sequencing jobs to minimize total weighted completion time subject to precedence constraints, Annals of Discrete Mathematics 2 (1978) 75-90.

[17] R. Chandrasekaran, S.N. Kabadi, Pseudomatroids, Discrete Mathematics 71 (1988) 205-217.

[18] U. Faigle, W. Kern, Submodular linear programs on forests, Mathematical Programming 72 (1996) 195-206.

[19] A. Frank, É. Tardos, Generalized polymatroids, Mathematical Programming 42 (1988) 489-563. 